\title{
DENSITÀ ELETTRONICA IN UNA IONOSFERA NON ISOTERMA
}

\author{
F. Mariani
}

Nella presente nota, studiamo (paragr. 1 e 2), quale completamento della teoria della fotoionizzazione in atmosfera non isoterma, considerata in altra nota (') che qui indicheremo con la sigla $M$, l'effetto della curvatura terrestre sulla intensità di ionizzazione (e quindi sulla densità elettronica) nei due casi in cui il gradiente verticale di temperatura sia costante in tutto l'intervallo di quote considerato (caso questo studiato da Gledhill e Szendrei $\left({ }^{2}\right)$ solo in condizioni stazionarie e trascurando l'effetto della curvatura terrestre) $e$ in quello in cui il gradiente ha un valore costante e positivo con la quota fino a una quota $c$ e valore zero per quote superiori. Deduciamo inoltre (paragr. 3) delle espressioni approssimate per la densità elettronica stabilendone i limiti di validità e di applicabilità alla interpretazione dei dati sperimentali; stabiliamo infine (paragr. 4) una relazione di validità generale, nel caso stazionario e per un generico andamento della temperatura con la quota, tra densità elettronica, coefficiente di ricombinazione generalizzato, densità di materia e coefficiente di assorbimento della radiazione monocromatica ionizzante.

Paragrafo 1. - Facendo riferimento alla precedente nota $M$ e ammettendo che il coefficiente di assorbimento della radiazione ionizzante non dipenda dalla quota possiamo scrivere, nel caso in cui la temperatura cresca linearmente e indefinitamente con la quota con legge $T=T_{0}(1+p z)$ e il raggio della Terra sia infinito, la intensità di ionizzazione $I(z)$ espressa dalla $(M, 5)$ nella forma

$$
I(z)=I_{0} \exp \left\{u\left[1-\sec x\left(\frac{1+p z}{1+p z^{*}}\right)^{\mathrm{r}}\right]\right\} \cdot\left(\frac{1+p z}{1+p z^{*}}\right)^{\mathrm{r}-1}
$$

ove

$I_{0}=\beta h S_{\infty} \exp [-\mu] \cdot\left(1+p z^{*}\right)^{\mathrm{r}-1}$ è il valore massimo di $I(z)$ allorché $\chi=0^{\circ}$, conseguito alla quota $z^{*}$;

$\beta=$ numero di ioni prodotti per l'assorbimento dell'unità di quantità di radiazione; 
$h=A_{\mathrm{o}} \rho_{\mathrm{o}}$, essendo $A_{\mathrm{o}}$ il coefficiente di assorbimento della radiazione e $\rho_{0}$ la densità di materia alla quota $z=0$;

$S_{\infty}=$ intensità della radiazione fuori della atmosfera;

$\mu=1+p H_{o}$, con $H_{0}=\frac{k T_{o}}{m g}$ scala delle altezze alla quota $z=0$ a cui la temperatura ha il valore $T_{0}$;

$$
\begin{aligned}
& r=-\frac{1}{p H_{0}}=-\frac{m g}{k \operatorname{grad} T} ; \\
& m=\text { massa molecolare media; } \\
& k=\text { costante di Boltzmann. }
\end{aligned}
$$

Se si introduce la variabile

$$
v=\frac{1+p z}{1+p z^{*}}
$$

la [1] viene a scriversi nella più semplice forma

$$
I(v)=I_{o} \exp \left[\mu\left(1-\sec \chi v^{r}\right)\right] \cdot v^{\mathrm{r}-1} \quad \text { per } v \geq \frac{1}{1+p z^{*}}
$$

Nel caso che si consirleri invece il raggio $R$ della Terra come finito la [3] viene sostituita, a partire dalla $(M, 39)$, dalla espressione

$$
I(v)=I_{\mathrm{o}} \exp [\mu+h g(v)] \cdot v^{\mathrm{r}-1}
$$

con

$$
g(v)=\int_{\infty}^{\gamma(v)}(1+p \xi)^{r-1} \cdot\left[1-\left(\frac{R+z(v)}{R+\xi}\right)^{2} \operatorname{sen}^{2} \chi\right]^{--1 / 2} d \xi
$$

Ponendo

$$
\left\{\begin{array}{l}
1+p \xi=a+b \cosh \tau \\
a=1-p R \\
b=\left[\left(1+p z^{*}\right) v-a\right] \operatorname{sen} \%
\end{array}\right.
$$

I"integrale [5] diviene

$$
g(v)=\frac{b}{p} \int_{\infty}^{\tau(v)}(a+b \cos h \tau)^{\mathrm{r}-1} \cos h-d \tau
$$




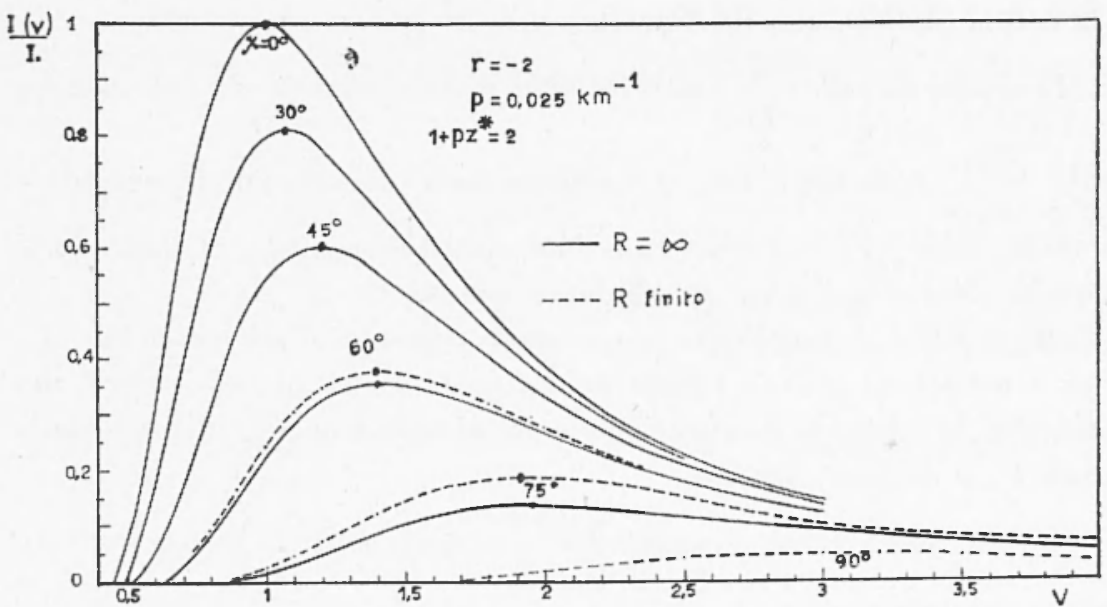

Fig. 1

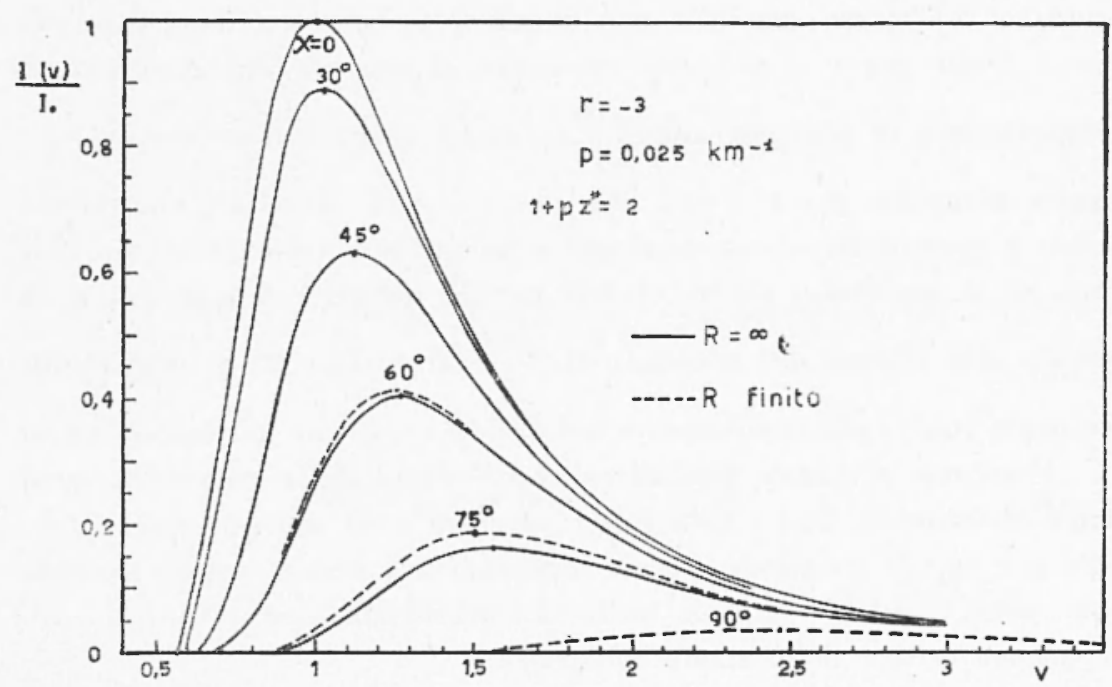

Fig. 2

Tale integrale è, per $r$ intero, calcolabile elementarmente: nclle figg. 1 e 2 riportiamo gli andamenti di $\frac{I(v)}{I_{o}}$, calcolati a partire dalle [3] e [4] per due valori di $r, r=-2$ e $r=-3$, rispettivamente corrispondenti ai valori $\frac{m g}{2 k}$ e $\frac{m g}{3 k}$ del gradiente di temperatura, cioè per es., assumendo $g=900 \mathrm{~cm} / \mathrm{sec}^{2}, m=25 \cdot 10^{-24} \mathrm{gr}$, ai gradienti 
8,22 e $5,47^{\circ} \mathrm{K} / \mathrm{km}$, rispettivamente; occorre notare che mentre la [3] dà l'andamento di $\frac{I(v)}{I_{o}}$ in funzione della variabile $v$, nel calcolo: della $\frac{I(v)}{l_{0}}$ a partire dalla [4] occorre anche fissare, per la seconda e la terza delle [6], il valore di $p$ che abbiamo assunto $=0,025 \mathrm{~km}^{-1}$ e quello di $1+p z^{*}$ che noi abbiamo assunto $=2$.

In quanto al raggio $R$ esso è stato posto, nel calcolo della [4], pari a $6600 \mathrm{~km}$, avendo tenuto conto, nella scelta di tale valore, del fatto che la quota alla quale inizia la distribuzione di densità dello strato $F_{1}$ è di circa $200 \mathrm{~km}$.

Si nota che i valori massimi di $\frac{I(n)}{I_{0}}$ sono, a parità di angrolo zenitale, sistematicamente più alti per $r=-3$ che per $r=-2$ nel caso $R=\infty$; nel caso $R$ finito, invece, l'effetto della curvatura terrestre comincia ad essere sensibile per angoli $\chi>60^{\circ}$ ed $\dot{e}$ maggiore per $r=-2$ che per $r=-3$ cioè crescente al crescere del gradiente di temperatura; in più, per angoli $\chi$ prossimi a $90^{\circ}$ il massimo di $\frac{I(,)}{I_{0}}$ risulta maggiore per $r=-2$ che per $r=-3$. Risultati qualitativamente e quantitativamente analoghi (che qui non riportiamo) si ottengono se si assumono valori diversi per le quantità $1+p z^{*}$ e $p$. In quanto alla altezza del massimo di $\frac{I(v)}{I_{\mathrm{o}}}$ al variare di $\%$ essa risulta crescente con $\%$, più rapidamente nel caso $R=\infty$ che nel caso $R$ finito.

Possiamo pertanto concludere che l'effetto della curvatura terrestre è trascurabile fino a valori di $\%$ prossimi a $60^{\circ}$ ma può essere notevole per angoli maggiori e, come vedremo nel paragr. seguente. notevole potrà risultare l'effetto sulla densità elettronica e quindi sulla frequenza critica dello strato ionizzato.

In modo del tutto analogo si esegue il calcolo della intensità di ionizzazione nel caso in cui si abbia

$$
\begin{cases}T=T_{0}(1+p z) & \text { per } z \leqslant c \\ T=T_{1}=T_{\mathrm{o}}(1+p c) & \text { per } z \geqslant c\end{cases}
$$

Le [3] e [4], a partire dalle $(M, 29)$ e $(M, 15)$, sono allora sostituite per il caso $R=\infty$ dalle espressioni

$$
I(v)=I_{0} \exp \left[\mu\left(1-\sec \chi v^{r}\right)\right] \cdot v^{r-1} \quad \text { per } \frac{1}{1+p z^{*}} \leq *<\frac{1+p c}{1+p z^{*}}=s
$$


$I(Z)=I_{0} \exp \left\{\mu\left[1-\sec \times s^{r} \exp (-Z)\right]-Z\right\} \cdot s^{r-1} \quad$ per $Z \geq 0$

ove con $Z$ si è indicata la variabile $Z=\frac{z-c}{(1+p c) H_{\mathrm{o}}}=\frac{z-c}{H_{c}}$ iò̀ la generica quota riferita alla quota $c$ e misurata in unità $H_{\mathrm{c}}=(1+p c) H_{0}$, essendo $H_{\mathrm{c}}$ il valore costante della scala delle altezzc al di sopra della quota $c$ stessa.

Nel caso che $R$ sia finito, invece, a partire dalle $(M, 42)$ e $(M, 47)$, si possono scrivere le espressioni

$$
I(v)=I_{\mathrm{o}} \in x_{\rho}\left\{\mu+h G(v)-\frac{\mu s^{\mathrm{r}}}{H_{\mathrm{c}}} \delta(s)\right\} \cdot v^{\mathrm{r}-1}
$$

$$
\operatorname{per} \frac{1}{1+p z^{*}} \leq v \leq s
$$

con

$$
\begin{array}{r}
G(v)=\int_{c(s)}^{:(v)}(1+p \xi)^{r-1}\left[1-\left(\frac{R+z(v)}{R+\xi}\right)^{2} \operatorname{sen}^{2} \chi\right]^{-1 / 2} d \xi \\
\delta(s)=\int_{c(s)}^{\infty} \exp \left(-\frac{\xi-c}{H_{c}}\right) \cdot\left[1-\left(\frac{R+c(s)}{R+\xi}\right)^{2} \operatorname{sen}^{-} \chi\right]^{--1 / 2} d \xi
\end{array}
$$

e

$$
I(Z)=I_{\mathrm{o}} \exp \left\{\mu\left[1-\frac{s^{\mathrm{r}}}{H_{\mathrm{c}}} \lambda(Z)\right]\right\} \cdot \mathrm{s}^{\mathrm{r}-1}
$$

$$
\text { per } Z \geq 0
$$

con

$$
\lambda(Z)=\int_{\mathrm{z}(\mathrm{Z})}^{\infty} \exp \left(-\frac{\xi-c}{H_{r}}\right) \cdot\left[1-\left(\frac{R+z(Z)}{R+\xi}\right)^{2} \operatorname{sen}^{2} \chi\right]^{-1 / 2} d \xi\left[13^{\prime}\right]
$$

I risultati del calcolo numerico sono esposti nelle figure 3 e 4 che danno gli andamenti di $\frac{I}{I_{0}}$ per vari va ri di $\chi$, in funzione delle variabili $v$ e $Z$ nei casi $1+p z^{*}=2, s=4$ e $1+p z^{*}=6, s=1,333$ e sempre per $p=0,025 \mathrm{~km}^{-1}$. 
Si vede dalla fig. 3 che, come era prevedibile, l'andamento di $\frac{I}{I_{0}}$ è praticamente coincidente con quello ottenuto nel caso della fig. 2 allorché la quota alla quale la $\frac{I}{I_{\mathrm{o}}}$ è massima per $\chi=0^{\circ}$ è assai più bassa della quota $c$. Nel secondo caso, invece, si constata la presenza

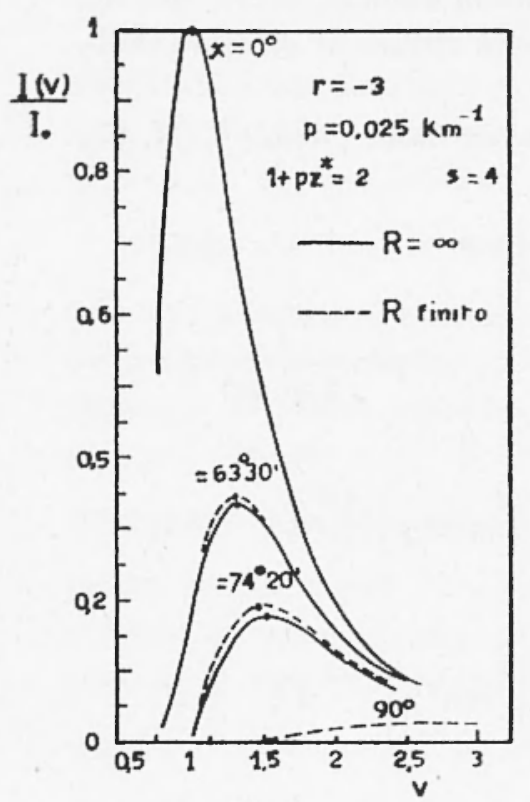

Fig. 3

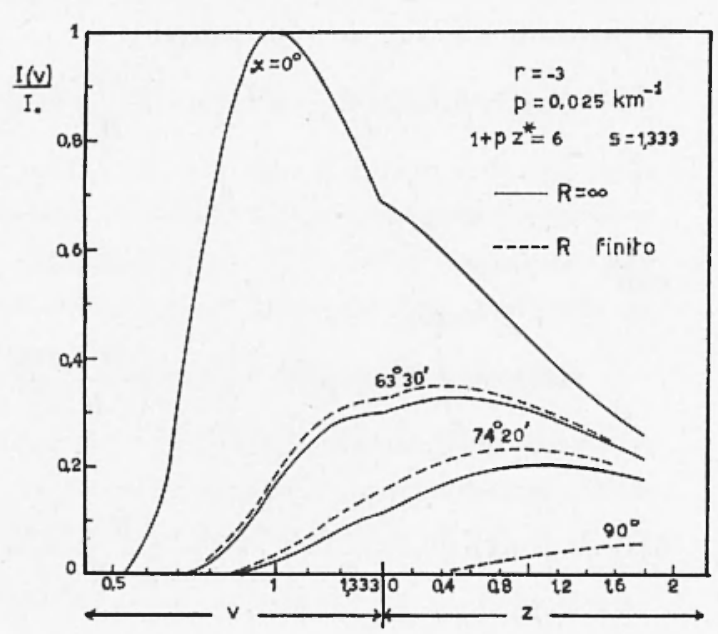

Fig. 4

di una discontinuità del gradiente della intensità $\frac{I}{I_{0}}$, di entità crescente al crescere dell'altezza del Sole sull'orizzonte cioè al diminuire dell'angolo zenitale. Per angoli $\chi$ maggiori di circa $75^{\circ}$ la discontinuità è praticamente scomparsa.

Gli andamenti di $\frac{I}{I_{0}}$ per altri valori dei parametri sono sempre del tipo indicato nelle figg. 3 e 4 sia per quanto riguarda la influenza della discontinuità del gradiente di temperatura che per l'effetto della curvatura terrestre.

Concludiamo allora, in generale, che anche nel caso in cui si abbia una discontinuità del gradiente verticale l'effetto della curvatura terrestre si fa sentire per $\chi \geq 60^{\circ}$ e quindi, essenzialmente, nelle prime 
c nelle ultime ore del giorno durante l'estate ma anche nelle ore meridiane durante l'inverno (a medie latitudini).

Paragrafo 2. - Applicheremo ora i risultati del paragrafo precedente al calcolo della densità elettronica. Considereremo la classica equazione

$$
\frac{d N}{d t}=I-\alpha N^{2}
$$

per la cui integrazione occorrerà fare delle ulteriori ipotesi sulla dipendenza di $\alpha$ dalla quota (o da altre variabili) oltreché sulla dipendenza della temperatura dal tempo.

Questi argomenti sono più ampiamente trattati in altro lavoro in preparazione; qui ci limiteremo a fare delle ipotesi di lavoro in quanto nostro scopo è quello di valutare l'ordine di grandezza delle correzioni che vanno apportate alla densità elettronica allorché si considera l'influenza della curvatura terrestre oltreché il caso non stazionario; l'interesse per queste due questioni è accresciuto dal fatto che l'effetto della prima è maggiormente sensibile proprio quando non sono valide, almeno a priori, le condizioni stazionarie studiate da Gledhill e Szendrei per il caso $R=\infty$.

Supporremo allora che il coefficiente di ricombinazione $\alpha$ si possa ritenere costante e che la temperatura sia, a ciascuna quota, costante nel tempo. La [14] si può particolarizzare nella forma

$$
\sigma \frac{d \nu}{d \Phi}=\frac{I}{I_{\mathrm{o}}}-\nu^{2}
$$

con

$$
\begin{aligned}
& \Phi:=\frac{2 \pi}{86400} t=\frac{t}{1,37 \cdot 10^{4}} \\
& \nu=\frac{N}{N_{\mathrm{o}}} \\
& N_{\mathrm{o}}=\sqrt{\frac{I_{\mathrm{o}}}{\alpha}} \\
& \sigma=\frac{1}{1,37 \cdot 10^{4} N_{\mathrm{o}} \alpha}
\end{aligned}
$$

La [15] è stata integrata numericamente con il metodo di Millington $\left({ }^{3}\right)$, con la condizione che la $\vee(\Phi)$ fosse. a ciascuna quota $v$, periodica in $\Phi$ con periodo $2 \pi$. Le figg. 5 e 6 mostrano l'andamento di 
$\checkmark(\Phi)$ per vari valori di $v$ ottenuto, rispettivamente, per un giorno al solstizio estivo e per un giorno al solstizio invernale alla latitudine di $45^{\circ}$ e per i valori $r=-2, p=0,025 \mathrm{~km}^{1}, 1+p z^{*}=2$ e $\sigma=\frac{1}{5}$

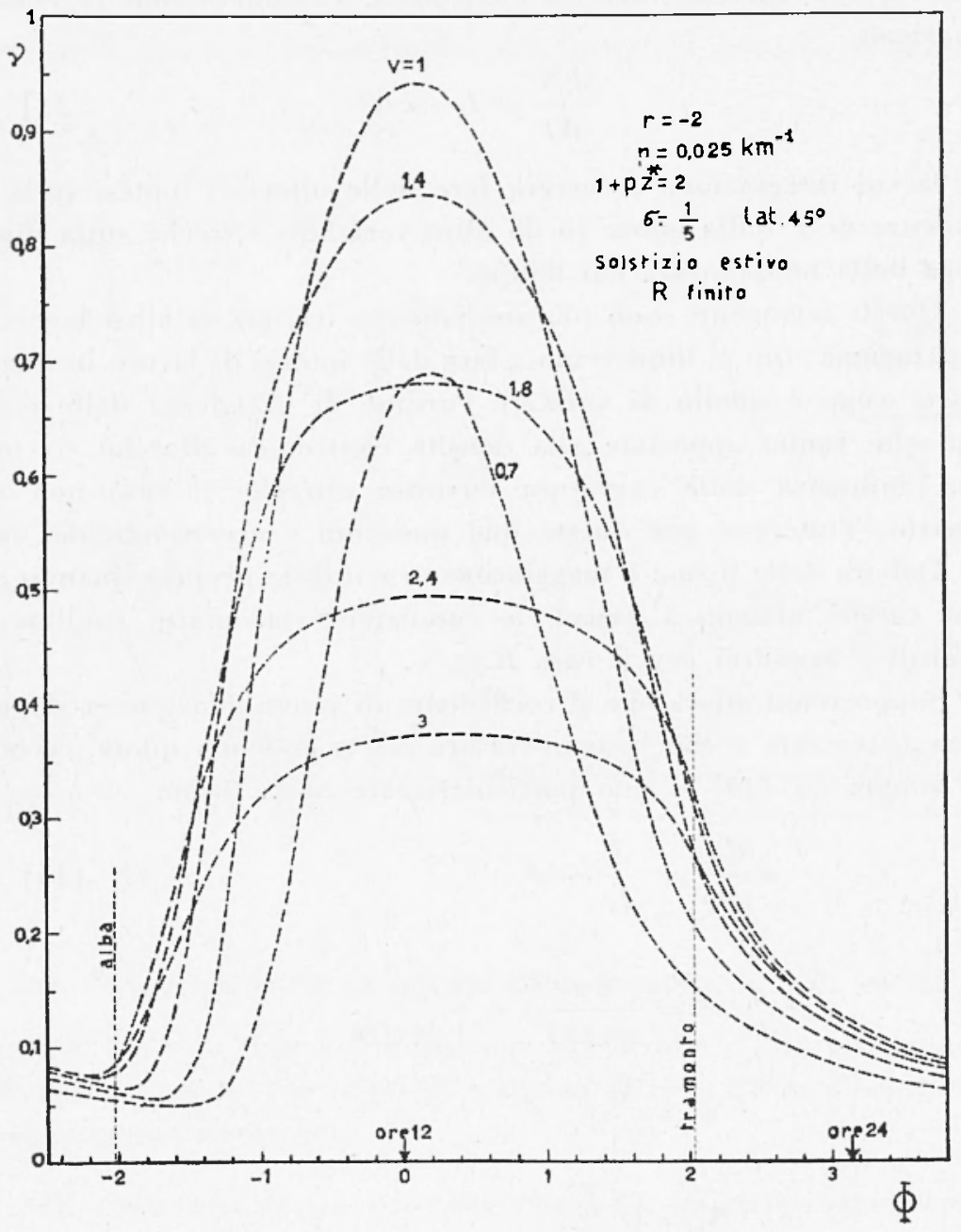

Fig. 5

assumendo una temperatura linearmente e indefinitamente crescente con la quota. Per il giorno estivo abbiamo riportato l'andamento di $v(\Phi)$ nel solo caso $R$ finito, in quanto piuttosto modeste sono le differenze rispetto al caso $R=\infty$ tranne nelle prime ore dopo l'alba. Sensibili risultano invece le differenze tra $i$ due casi nel giorno invernale. 
Dalle curve graficate è possibile dedurre l'andamento di $\vee(v)$ istante per istante: nella fig. 7 riportiamo, come più rispondenti al nostro scopo, gli andamenti orari di $\left(\nu_{\max }\right)^{1 / 2}$ che sono proporzionali alle

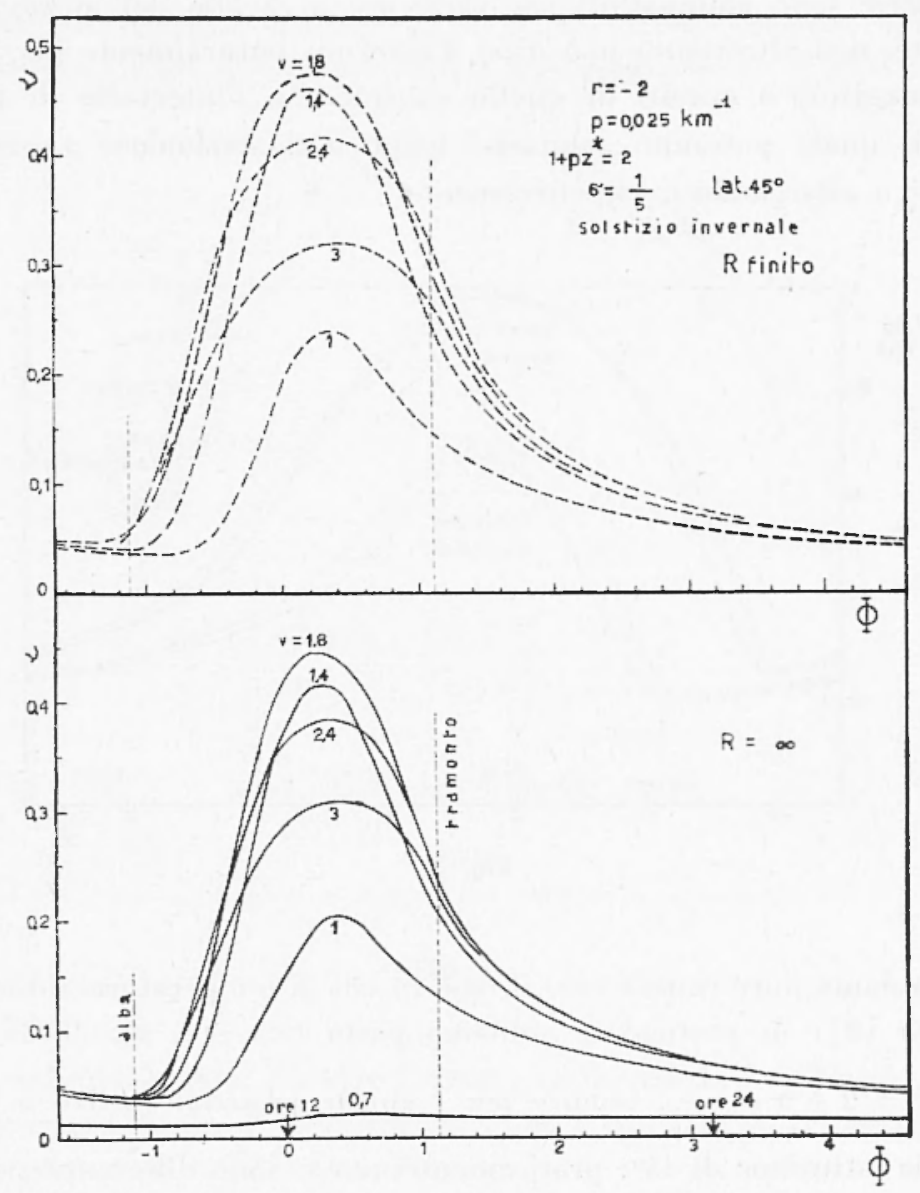

Fig. 6

frequenze critiche $f_{c}$, ottenuti nei due casi $R=\infty$ e $R$ finito per i due giorni tipici. Si nota come d'estate le maggiori differenze percentuali tra i due casi si manifestano qualche ora dopo l'alba (fino al 25\%) $e$, con minore entità, verso il tramonto mentre nelle ore meridiane l'andamento di $f_{c}$ è praticamente lo stesso; d'inverno, invece, le differenze sono assai più marcate e sussistono anche nelle ore meridiane. 
In quanto all'altezza del massimo di densità elettronica (che non riportiamo) essa risulta minima e piuttosto simmetrica intorno al mezzogiorno. Nella stessa fig. 7 sono anche riportati punteggiati gli andamenti di $\left(v_{\max }\right)^{1 / 2}$ per il caso stazionario; si constata che se le condizioni stazionarie sono ammissibili per varie ore a cavallo del mezzogiorno in estate, non altrettanto può dirsi d'inverno; naturalmente per valorì di $\sigma$ maggiori o minori di quello considerato, l'intervallo di tempo entro il quale potranno assumersi condizioni stazionarie va restringendosi o allargandosi, rispettivamente.

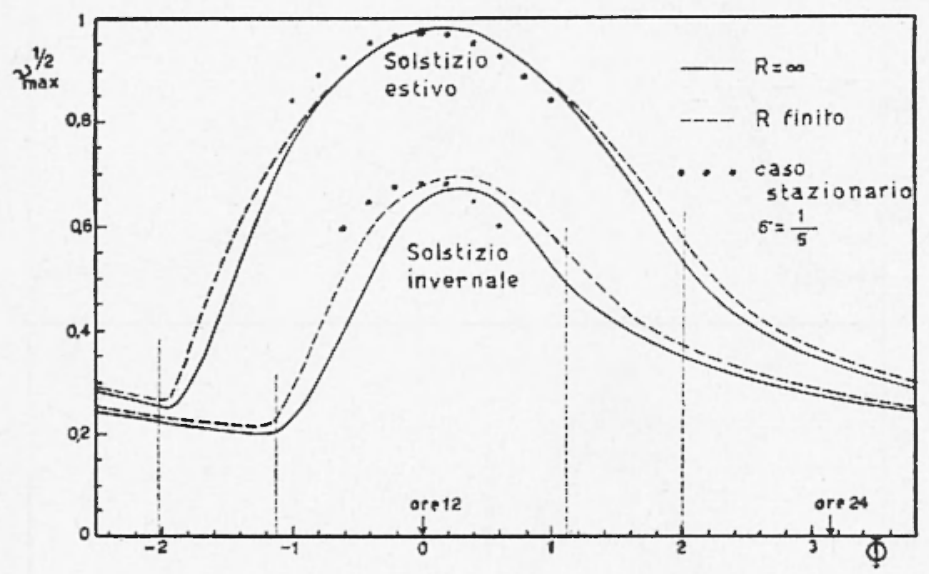

Fig. 7

Abbiamo pure considerato il caso in cui la temperatura abbia l'an. damento [8]: in particolare abbiamo posto $r=-2, p=0,025 \mathrm{~km}^{-1}$, $1+p z^{*}=2$ e $\sigma=\frac{1}{5}$, sempre per i giorni solstiziale estivo e invernale alla latitudine di $45^{\circ}$ : praticamente non vi sono differenze rispetto agli andamenti $d i\left(v_{\max }\right)^{1 / 2}$ mostrati nella fig. 7 .

Fin qui si è considerata l'eventualità che il coefficiente di ricom. binazione $\alpha$ fosse costante; ove si volesse considerarne la diminuzione con la quota, pur avendosi naturalmente degli andamenti di densità e quindi di frequenza critica diversi a seconda della legge di dipendenza di $\alpha$ dalla quota (o da altre variabili), si possono fare delle considerazioni qualitative; sia $\alpha_{\text {c }}$ il valore di $\alpha$ alla quota fissa $c$ e ammettiamo che sia $\alpha>\alpha_{c}$ a quote inferiori a $c$ e $\alpha<\alpha_{c}$ a quote superiori: conseguenza di ciò è un innalzamento sistematico del massimo 
di densità elettronica e, se la variazione di $\propto$ con la quota è suffcientemente rapida, tale massimo verrà a trovarsi sempre al di sopra della quota $c$ : in questo caso, alla quota $c$, si avrebbe una discontinuità del grad $\vee$ cui, da un punto di vista sperimentale, verrebbe a corrispondere una discontinuità della curva $h^{\prime}(f)$ dell'altezza virtuale. Ciò premesso si può notare che effettivamente una discontinuità $h^{\prime}(f)$ si riscon.

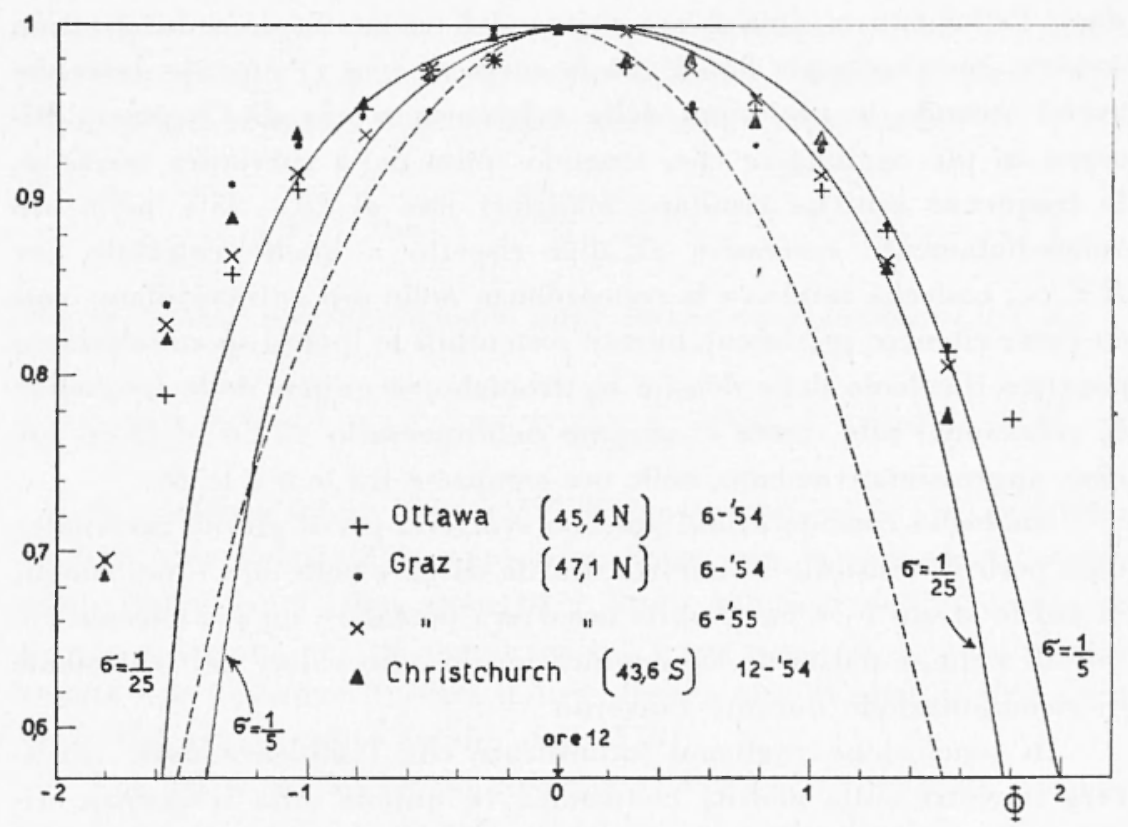

Fig. 8

tra, a medie latitudini, durante l'estate: la frequenza $f$ dell'onda riflessa in corrispondenza di tale discontinuità è quella che si chiama frequenza critica $f_{0} F_{1}$ dello strato $F_{1}$.

Consideriamo la fig. 8, nella cui preparazione ciascuna serie di valori numerici indicati, sia calcolati sia sperimentali, è stata moltiplicata per opportuni coefficienti di normalizzazione allo scopo di renderli tutti uguali all'unità in corrispondenza del mezzogiorno così da favorirne il confronto senza alterarne il significato fisico: le linee continue indicano gli andamenti delle frequenze di riflessione alla quota $c$, calcolati per due valori di $\sigma$ e per $\boldsymbol{R}=\infty$, la linea tratteggiata dà l'andamento della funzione $(\cos \chi)^{1 / 2}$ mentre $\mathrm{i}$ vari tipi di punti indicano $\mathrm{i}$ valori mediani mensili di $f_{0} F_{1}$ ricavati dai dati sperimentali ottenuti 
nel mese del solstizio estivo in vari osservatori situati a latitudini assai prossime ai $45^{\circ}$, nei quali l'alba e il tramonto si hanno rispettivamente intorno alle 4 e alle 20 , ovvero in termini di $\Phi$, per $\Phi \simeq-2$ e $\simeq+2$. Si nota una sensibile concordanza, maggiore nel caso $\sigma=\frac{1}{25}$ che nel caso $\sigma=\frac{1}{5}$, tra dati sperimentali e valori calcolati, da circa 4 ore dopo l'alba fino a circa 2 ore prima del tramonto, accordo che non sussiste con altrettanta bontà con la curva di $(\cos \chi)^{1 / 2}$ quale dovrebbe aversi secondo le previsioni della originaria teoria di Chapman. Bisogna in più aggiungere che, tenendo conto della curvatura terrestre, le frequenze critiche risultano maggiori fino al $20 \div 25 \%$ nelle ore immediatamente successive all'alba rispetto a quelle calcolate per $\boldsymbol{R}=\infty$, cosicché migliora la concordanza nelle ore antimeridiane, cosi da poter ritenere sperimentalmente sostenibili le ipotesi su cui abbiamo poggiato il calcolo delle densità elettroniche (e quindi delle frequenze di riflessione) alla quota $c$, almeno nell'intervallo $-1,6<\Phi<1,6$, cioè, approssimativamente, nelle ore comprese tra le 6 e le 18 .

Analoghe considerazioni possono svolgersi per il giorno invernale: data però la sensibile simmetria diurna di $f_{0} F_{1}$ nelle ore e nei luoghi in cui lo strato $F_{1}$ è osservabile occorrerà postulare un più piccolo valore di $\sigma$ che, a parità di $N_{0}$, significa un più alto valore del coefficientc di ricombinazione durante l'inverno.

In conclusione vogliamo sottolineare che l'influenza della curvatura terrestre sulla densità elettronica (e quindi sulla frequenza critia) non va sottovalutata in quanto essa può essere piuttosto sensibile e ciò in particolare quando si vogliano studiare i fenomeni che vengono al mattino o al tramonto per i quali appare senz'altro non lecito fare a meno di considerarla.

In più l'apprezzabile concordanza tra valori sperimentali di $f_{0} \boldsymbol{F}_{1}$ e valori calcolati sopra rilevata, può apparire incoraggiante cosi da suggerire la possibilità di riesaminare più in dettaglio, sulla base della [8] e tenendo nel dovuto conto la diminuzione con la quota del coefficiente di ricombinazione, tutta la questione della regione $F$ nel suo complesso; ma per tale argomento rinviamo a una prossima nota ora in preparazione.

Paragrafo 3. - In questo paragrafo vogliamo stabilire i limiti di validità di talune approssimazioni assai frequenti nella riduzione delle curve $h^{\prime}(f)$ alle altezze vere; il caso più spesso considerato è quello 
della approssimazione parabolica per il quale la densità elettronica viene supposta funzione quadratica della quota. Ci limiteremo, per semplicità di trattazione, al caso stazionario e al caso $R=\infty$ : dalle [15] e [3] si deduce immediatamente

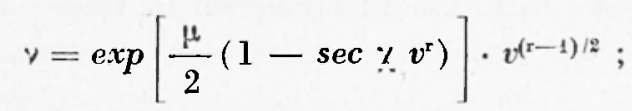

indicando con $v_{\mathrm{m}}$ il valore di $v$ in corrispondenza del quale y è massima, sviluppando la $\vee$ stessa in serie di Taylor intorno al punto $v_{m} \mathrm{e}$ arrestandoci al termine quadratico otteniamo l'espressione

$$
\nu(v)=\nu_{\max }\left[1+\frac{r(1-r)}{4} \sec \chi^{2 / r}\left(v-v_{m}\right)^{2}\right]
$$

che si traduce immediatamente nella forma equivalente, in $z$ e $z_{\mathrm{n} \text {, }}$

$$
\nu(z)=\nu_{\mathrm{rax}}\left[1+\frac{r(1-r)}{4} \frac{p^{2}}{\left(1+p z^{*}\right)^{! !}} \sec \chi^{2 / \mathrm{r}}\left(z-z_{\mathrm{m}}\right)^{2}\right]
$$

Si stabilisce subito l'intervallo di quote entro cui la [16] può identificarsi, con determinata approssimazione, con le precedenti espressioni. Nella fig. 9 sono riportati i limiti inferiori dell'intervallo di $v-v_{\mathrm{m}}$ entro cui, ai vari angoli zenitali e per $r=-2$, la $v(v)$ approssimata non differisce di oltre il $10 \%$ (curva a) e di oltre il $50 \%$ (curva $b$ ) dal valore esatto fornito dalla [16].

Fin qui il coefficiente di ricombinazione è stato supposto costante con la quota; se ora ammettiamo, per es., che esso dipenda dalla quota $v$ secondo la legge $\alpha \sim \frac{1}{v^{\gamma}}$ le $[16],[17],[18]$ sono sostituite dalle espressioni

$$
\begin{gathered}
\nu(v)=\exp \left[\frac{\mu}{2}\left(1-\sec \chi v^{\mathrm{r}}\right)\right] \cdot v^{(\mathrm{r}-1+\gamma) / 2}, \quad[19] \\
\nu(v)=\nu_{\max }\left[1+\frac{r(1-r-\gamma)}{4}\left(\frac{1-r-\gamma}{1-r}\right)^{-2 / \mathrm{r}} \sec \chi^{i / r}\left(v-v_{\mathrm{m}}\right)^{s}\right]
\end{gathered}
$$

$\nu(z)=\psi_{\mathrm{max}}\left[1+\frac{r(1-r-\gamma)}{4}\left(\frac{1-r-\gamma}{1-r}\right)^{-2 / \mathrm{r}} \frac{p^{2}}{\left(1+p z^{*}\right)^{2}} \sec \chi^{2 / \mathrm{r}}\left(z-z_{\mathrm{m}}\right)^{2}\right]$.

Ancora nella fig. 9 è riportato il limite inferiore dell'intervallo di $v-v_{m}$ entro cui, supposto $\gamma=2$, la espressione quadratica appros- 
simata di $v(v)$ non differisce di oltre il $10 \%$ (curva $c$ ) e di oltre il $50 \%$ (curva $d$ ) dal valore esatto fornito dalla [19]. Si constata che il limite inferiore di $v-v_{\text {m }}$ è maggiore nel caso $\alpha \sim \frac{1}{v^{2}}$ che nel caso $\alpha=$ cost e che, in ambedue $i$ casi, esso va crescendo in valore al crescere del. l'angolo zenitale.

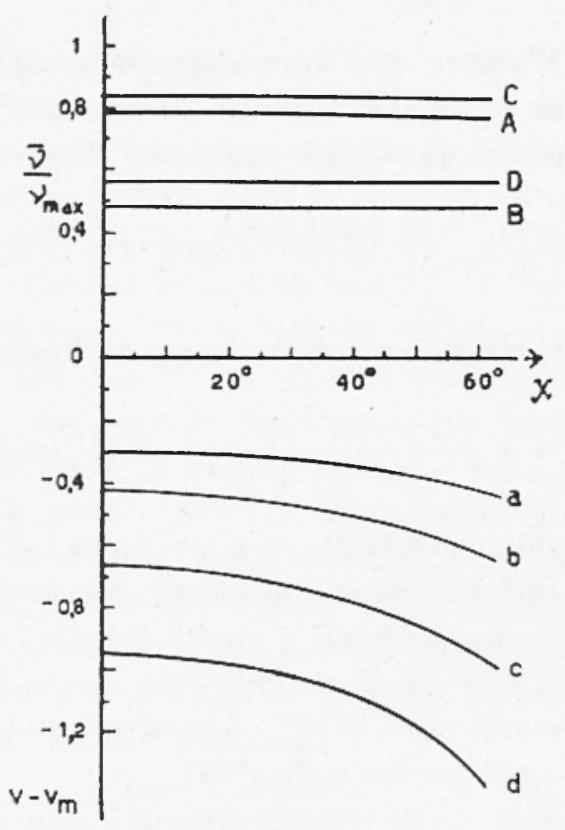

Fig. 9

Più interessanti risultano le curve $A, B, C, D$, che mostrano la dipendenza dall'angolo zenitale del rapporto $\bar{v} / v_{\operatorname{maz}}$ tra la densità esatta $\bar{v}$ alle quote rappresentate rispettivamente dalle curve $a, b, c, d$ e la densità massima $v_{\text {max }}$ : si nota che, anche nel caso della curva $B$, tale rapporto è piuttosto alto $(\sim 0,48)$ e che, in ogni caso, esso non dipende sensibilmente da $\%:$ ne consegue, in generale, che le frequenze di riflessione alle quote per le quali l'approssimazione quadratica può considerarsi accettabile risultano non inferiori al $70 \div 30 \%$ della frequenza critica. Dal punto di vista sperimentale ciò equivale a dire che l'intervallo di quote entro cui è valida la approssimazione quadratica per una atmosfera in cui la temperatura cresce linearmente e indefinitivamente con la quota viene a corrispondere un intervallo di frequenze riflesse sensibilmente prossime alla frequenza critica: ma per tali fre. 
quenze la altezza virtuale $h^{\prime}(f)$ è una funzione rapidamente crescente di $f$ e gli errori sperimentali di lettura sugli ionogrammi possono essere rilevanti.

Ne concludiamo che ampie riserve vanno fatte, almeno nelle condizioni studiate, ai risultati ottenuti nella interpretazione dei dati sperimentali basati nell'uso dei modelli “parabolici ” per la distribuzione di densità elettronica, risultati che vanno considerati come qualitativi e quindi necessitanti di più attento controllo e più precisa conferma per essere accettati.

Paragrafo 4. - Vogliamo da ultimo esaminare un'altra questione; consideriamo la più generale espressione per la intensità di ionizzazione $I(z)$ che, ponendo $y(z)=A(z) \rho(z)$, ove $A(z)$ è il coefficiente di assorbimento della radiazione ionizzante (che in tutto ciò che precede abbiamo supposto costante) e $\rho$ (z) la densità di materia, si scrive per le $(M, 5)$ e $(M, 3)$ :

$$
I(z)=\beta S_{\infty} y \exp \left[\sec \chi \int_{\infty}^{\mathrm{z}} y \bar{a} z\right] .
$$

In condizioni stazionarie vale allora l'equazione

$$
\beta S_{\infty} y \exp \left[\sec \chi \int_{\infty}^{\mathrm{z}} y d z\right]=\alpha N^{\circ}
$$

Derivando, rispetto a $z$, membro a membro la [23] e quindi dividendo ancora membro a membro per la [23] stessa, si perviene, con facili passaggi, all'equazione

$$
\frac{d y}{d z}=P y-Q y z
$$

con

$$
\left\{\begin{array}{l}
P=\frac{1}{\alpha N^{2}} \frac{d\left(\alpha N^{\circ}\right)}{d z} \\
Q=\sec \%
\end{array}\right.
$$

Ponendo ancora

$$
y-\cos \chi \frac{1}{u} \frac{d u}{d z}
$$


la [24] si scrive nella forma

$$
\frac{d^{2} u}{d z^{2}}-P \frac{d u}{d z}=0
$$

il cui integrale generale è

$$
u(z)=\delta_{1} \int_{0}^{\mathrm{z}} \alpha N^{2} d z+\delta_{2}
$$

$\operatorname{con} \delta_{1}$ e $\hat{o}_{0}$ costanti arbitrarie.

In conclusione, dalla [26] deduciamo la più generale soluzione della [24], dipendente da una sola costante di integrazione $\delta$, nella forma

$$
y(z)=\cos \chi \frac{\alpha N^{2}}{\delta+\int_{0}^{z} \alpha N^{2} d z}
$$

La costante $\delta$ va determinata imponendo, per es., il valore $y_{0}$ al livello, peraltro arloitrario, $z=0$, cosi da ottenere l'espressione

$$
y(z)=y_{0} \frac{\alpha N^{2}}{\left(\alpha N^{2}\right)_{0}+y_{0} \sec \chi \int_{0}^{z} \alpha N^{2} d z}
$$

La [30] è formula di validità generale nel caso stazionario e per $R=\infty$ e la sua applicazione si semplifica ovviamente nei casi particolari, per es. quando si assuma $A(z)=A_{0}=$ cost. Si constata immediatamente che da essa è possibile dedurre una espressione di $\alpha(z)$ che viene a coincidere con quella che si deduce dalla [23] imponendo la condizione $\alpha=\alpha_{0}$ per $z=0$; si ha precisamente

$$
\alpha(z)=\alpha_{0} \frac{y}{N^{2}} \cdot\left(\frac{N^{2}}{y}\right)_{0} \exp \left[\sec \chi \int_{0}^{\mathrm{z}} y d z\right] .
$$

La utilità delle [30] e [31] può essere limitata dalle incertezze relative alle grandezze che in essa intervengono, oltreché dall'essere 
dedotte nel caso stazionario e nell'ipotesi che la radiazione ionizzante sia monocromatica; a titolo di esempio applicheremo tali formule a un caso in cui la densità $\nu$ cresce linearmente con la quota con la legge

$$
\nu=\nu_{\mathrm{o}}(1+q z)
$$

Tale andamento di $v$ approssima abbastanza bene per es. quello sperimentale determinato con rocket da Seddon, Pickar e Jackson $\left({ }^{4}\right)$ : dalla fig. 8 riportata da tali Autori risulta che la densità elettronica cresce appunto più o meno linearmente con la quota nell'intervallo tra 100 e $160 \mathrm{~km}$ dal suolo.

Nella fig. 10 riportiamo (curva a) l'andamento di $\log _{10}\left[\rho(z) / \rho_{0}\right]$

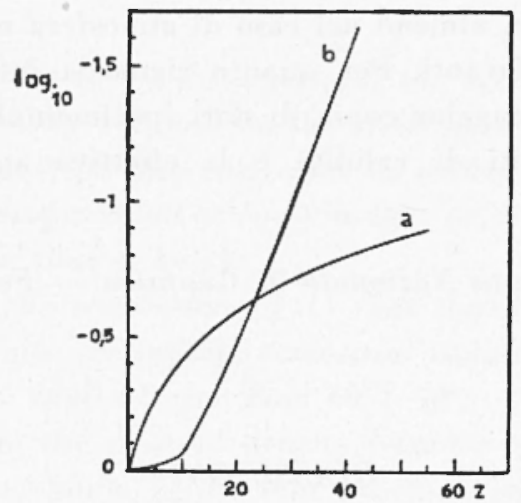

Fig. 10

dedotto dalla [30] nel caso in cui sia $q=10^{-7} \mathrm{~km}^{-1} ; A=A_{0}=$ cost $=$ $10^{-18} \mathrm{~cm}^{2} ; \rho_{\mathrm{o}}=10^{-9} \mathrm{gr} / \mathrm{cm}^{3}$ alla quota di $100 \mathrm{~km}$ dal suolo assunta come quota 0 nella [32]; $\chi=0^{\circ} ; \alpha=\alpha_{0}=$ cost ; la curva $b$ mostra invece l'andamento di $\log _{10}\left[\alpha(z) / \alpha_{0}\right]$ nel caso in cui sia

$$
P=\rho_{0} \exp \left(-z / z_{0}\right) \quad \text { con } z_{0}=8,7 \mathrm{~km}
$$

e la densità $v$ sia data sempre dalla [32] : appare ben evidente in questo caso una rapida diminuzione di $\alpha$ al crescere di $z$, con legge pressoché esponenziale tranne nei primi $\mathbf{k m}$ al disopra della quota 0 entro i quali $\alpha$ si mantiene pressoché costante.

Conclusioni. - I risultati conseguiti nel presente lavoro ci permettono di stabilire che l'effetto della curvatura terrestre sulla intensità di ionizzazione comincia ad essere apprezzabile per angoli zenitali 
prossimi a $60 \div 70^{\prime}$ e che notevole può anche essere l'effetto sulla densità elettronica (e quindi sulla frequenza critica in particolare) soprattutto ai crepuscoli e, d'inverno, a medie latitudini anche durante la giornata: naturalmente però leggi di dipendenza del coefficiente di ricombinazione dalla quota e dal tempo diverse da quelle qui considerate possono influire più o meno sensibilmente sugli andamenti quantitativi di $v$. Inoltre un risultato degno di rilievo è la concordanza rilevata tra i valori sperimentali di $f_{0} F_{1}$ (per un giorno solstiziale estivo alla latitudine di $45^{\circ}$ ) e i valori di $y^{1 / 2}$ alla quota $c$ calcolati nel modello di atmosfera che si basa su una distribuzione di temperatura con la quota del tipo [8]. In quanto alla approssimazione parabolica che spesso viene assunta per l'andamento della densità elettronica con la quota vanno fatte, almeno nel caso di atmosfera non isoterma, delle riserve piuttosto rilevanti. Per quanto riguarda infine $i$ risultati del parag. 4 solo una maggior copia di dati sperimentali potrà consentire di stabilirne i limiti di validità e la effettiva applicabilità a casi concreti.

$$
\text { Roma - Istituto Nazionale di Geofisica - Febbraio } 1955 .
$$

\section{RIASSUNTO}

Nella presente nota si studia la dipendenza della ionizzazione $\mathrm{I}(\mathrm{z})$ e della densità elettronica $\mathbf{N}(\mathrm{z})$ dalla curvatura terrestre in una atmosfera non isoterma nei due casi in cui il gradiente verticale di temperatura sia a) costante a ogni quota oppure b) da una certa quota c in su assuma valore nullo; in ambedue $i$ casi le differenze rispetto al caso in cui la Terra sia supposta di raggio infinito cominciano ad apprezzarsi per angoli zenitali del Sole maggiori di $60 \div 70^{\circ}$ : si ha un aumento delle frequenze critiche in ogni caso massimo nelle ore centrate intorno all'alba $e, d^{\prime}$ 'inverno, piuttosto sensibile anche nelle altre ore della giornata.

Nel caso b si constata che la densità elettronica $\mathrm{N}$ alla quota c si accorda sensibilmente con $i$ valori sperimentali di $\left(\mathrm{f}_{0} \mathrm{~F}_{1}\right)^{2}$ assai puù che con $(\cos \chi)^{1 / 2}$ come previsto dalla originaria teoria di Chapman.

Si discutono quindi i limiti di validità della approssimazione "parabolica " per la densità elettronica $\mathrm{N}$ che risultano assai ristretti così 
da far ritenere poco attendibili le deduzioni basate su tale approssimazione, almeno nel caso di atmosfera non isoterma.

Infine si stabilisce una relazione generale, valida in condizioni stazionarie, tra temperatura, densità elettronica, coefficiente di ricombinazione generalizzato, densità di materia e coefficiente di assorbimento della radiazione (monocromatica) ionizzante, che può essere utile per confermare o per integrare, le informazioni sperimentali.

\section{$S U M M A R Y$}

In this paper we study the effects of the terrestrial curvature on the ionization intensity $\mathrm{I}(\mathrm{z})$ and on the electron density $\mathrm{N}(\mathrm{z})$, in a notisotherm atmosphere, for the two cases in which (i) the temperature gradient has a constant value at every height or (ii) assumes the value 0 at heights larger than a height $\mathrm{c}$.

Ire come to the conclusion (\$ 1) that the differences of $\mathrm{I}(\mathrm{z})$ in the two cases of finite or infinite terrestrial radius $\mathrm{R}$ grow rather important at zenithal angles larger than $60 \div 70^{\circ}$.

With regard to the electron density $\mathrm{N}(\mathrm{z})$ we consider also the not stationary case and show (\$2) that the differences in the cases of finite or infinite $\mathrm{R}$ may grow important expecially during the winter; in every case, if we assume a finite value of $\mathbf{R}$ the critical frequency of a layer after sunrise may assume values of $20-25 \%$ larger than in the case of infinite $\mathrm{R}$. The results obtained in the case of a temperature variable according to the scheme [8] exibit an interesting feature: the calculated electron density $\mathrm{N}_{\mathrm{c}}$ at the height c agrees with the experimental values of $\left(\mathrm{f}_{0} \mathrm{~F}_{1}\right)^{2}$ more closely than $(\cos \chi)^{1 / 2}$ as predicted by the Chapman theory. Hence, if we assume the height c as the separation of the $\mathrm{F}_{1}$ layer from the $\mathrm{F}_{2}$ layer, we may consider a tentative scheme of a composite $\mathrm{F}$ region based on the temperature distribution [8]; we study this question in another paper now in preparation.

We after consider (\$ 3) the "parabolic" approximation of the electron density $\mathrm{N}$ and show that, at least in the case of a not-isotherm atmosphere, it is not a good approximation; particularly we show that the approximation may considered acceptable only within the interval of heights corresponding to reflection frequencies not less than $0,7 \div 0,8$ 
times the critical frequency; but the experimental errors on the virtual height $\mathrm{h}^{\prime}(\mathrm{f})$ corresponding to these frequencies may be importunt so that the results of the interpretation of the ionograms based on the parabolic approximation may be affected by noticeable uncertainties.

At least we state (\$ 4) a general relation between temperature, electron density, generalized recombination coefficient, matter density and absorption coefficient of the ionizing radiation, valid, in the stationary conditions, for a monochromatic radiation. This relation may be useful, for example, in order to test or to integrate the experimental data; however further data are required in order to try its validity.

\section{BIBLIOGRAFIA}

(1) Mariani F., Ann. Geofis., 8-59 (1955).

(2) Gledhill J. A., Szendrei M. E., Proc. Phys. Soc. B, $63-427$ (1950).

(3) Millington G., Proc. Phys. Soc., 44-580 (1932).

(4) Seddon J. C., Pickar A. D., Jackson J. E., J. Geophys. Res., 59.513 (1954I. 